\title{
Current transport and loss mechanisms in the $Z$ accelerator
}

\author{
N. Bennett $\odot,{ }^{1}$ D. R. Welch $\odot,{ }^{2}$ C. A. Jennings, ${ }^{1}$ E. Yu, ${ }^{1}$ M. H. Hess, ${ }^{1}$ B. T. Hutselø ${ }^{1}$ \\ G. Laity, ${ }^{1}$ J. K. Moore, ${ }^{1}$ D. V. Rose, ${ }^{2}$ K. Peterson, ${ }^{1}$ and M. E. Cuneo ${ }^{1}$ \\ ${ }^{1}$ Sandia National Laboratories, Albuquerque, New Mexico 87185, USA \\ ${ }^{2}$ Voss Scientific, LLC, Albuquerque, New Mexico 87108, USA
}

(Received 24 July 2019; published 10 December 2019)

\begin{abstract}
A challenge for the TW-class accelerators driving Z-pinch experiments, such as Sandia National Laboratories' $Z$ machine, is to efficiently couple power from multiple storage banks into a single multi-MA transmission line. The physical processes that lead to current loss are identified in new large-scale, multidimensional simulations of the $Z$ machine. Kinetic models follow the range of physics occurring during a pulse, from vacuum pulse propagation to charged-particle emission and magnetically-insulated current flow to electrode plasma expansion. Simulations demonstrate that current is diverted from the load through a combination of standard transport (uninsulated charged-particle flows) and anomalous transport. Standard transport occurs in regions where the electrode current density is a few $10^{4}-10^{5} \mathrm{~A} / \mathrm{cm}^{2}$ and current is diverted from the load via transport without magnetic insulation. In regions with electrode current density $>10^{6} \mathrm{~A} / \mathrm{cm}^{2}$, electrode surface plasmas develop velocity-shear instabilities and a Hall-fieldrelated transport which scales with electron density and may, therefore, lead to increased current loss.
\end{abstract}

DOI: 10.1103/PhysRevAccelBeams.22.120401

\section{INTRODUCTION}

Experimental studies of dense Z-pinch implosions require magnetic fields of $100-1000 \mathrm{~T}$ generated by multi-MA currents within mm-diameter cylindrical geometries $[1,2]$. A challenge for the TW-class accelerators driving $Z$-pinch experiments is to efficiently couple power from multiple storage banks into a single multi-MA transmission line. The physical processes that lead to current loss derive from rapid heating of the electrode surfaces, which may generate charged particle emission undergoing transport without magnetic insulation and current-shunting plasma formations.

The accelerators addressing these issues include the $Z$ machine at Sandia National Laboratories [3,4], the Primary Test Stand (PTS) at the China Academy of Engineering Physics [5,6], and the MIG generator at the Institute of High Current Electronics $[7,8]$. Transmission-line-circuit models of $Z$ [9] and the PTS [10] have increased our understanding of the electrical coupling of the pulse-forming components to the transmission lines and the magnitude of power lost in transit to the load. Simultaneously, particle-in-cell (PIC) methods were advanced to model localized Joule heating and plasmas resulting from desorbed contaminants in MA-scale

Published by the American Physical Society under the terms of the Creative Commons Attribution 4.0 International license. Further distribution of this work must maintain attribution to the author(s) and the published article's title, journal citation, and DOI. transmission lines based on the $Z$ design [11]. It was found that higher rates of cathode plasma desorption decreased the effective transmission line gap and created conditions for current shunting away from the load.

In this paper, large-scale, 2 and 3-dimensional simulations are used to study the causes of power loss in the $Z$ machine, from its four radial power feeds through its current adder to the load. To encompass the radial feeds in realistic models of $Z$, the volumes in the simulation encompass $5 \times 10^{4} \mathrm{~cm}^{3}$, with field energy densities from a few $\mathrm{J} / \mathrm{cm}^{3}$ to nearly one $\mathrm{MJ} / \mathrm{cm}^{3}$ at the load. The simulations use a magnetic implicit particle and field solution $[12,13]$ which simultaneously relax the time constraints of the cyclotron and plasma frequencies, enabling $\omega_{c} \Delta t>1$ and $\omega_{p} \Delta t>1$. An adaptive time step and particle management [14] enable both the time step and macroparticle count to adjust to the plasma generated in the unified electrode plasma model [13]. With these algorithms and sufficient resolution, the simulations are able to span the range of physical processes that occur in a $Z$ shot, including vacuum pulse propagation, charged-particle emission, magnetically insulation current flow, and plasma formation and expansion.

The expected early-time behavior of the $Z$ machine is derived from studies of lower-energy accelerators $[15,16]$, in which the pulse propagation in vacuum creates sufficient electric field stresses and Joule heating to initiate spacecharge-limited electron emission [17] from the cathode. Electrons cross the anode-cathode (AK) gap and further heat the anode surface until their flow becomes magnetically 
insulated. Positive ions are drawn from the hot anode into either a counterstreaming or insulated current flow. The electrode plasmas from which the electrons and ions are drawn are created from desorbed surface contaminants, as modeled as in Refs. [11,13]. A realistic circuit model [9] provides the driving pulse so simulated currents may be validated against data.

The late-time current loss behavior of $Z$ operation is influenced by electrode plasmas with measured densities from $10^{16}$ to greater than $10^{17} \mathrm{~cm}^{-3}$ [18]. The electrode physics detailed in Ref. [13] creates conditions for dense current-carrying plasma formation and expansion into the AK gap. The impact of plasma expansion will be greatest at radii interior to the current adder, where the current densities and surface temperatures are highest. Although surface temperatures exceed melt in some locations, this transition is not considered here.

Details of the simulation geometries and the particle and field models that capture the early and late-time physics are provided in Sec. II. This technique is applied to four hardware geometries fielded on $Z$ in order to compare to data over multiple runs and to demonstrate the relationships between hardware geometry and power loss. As detailed in Ref. [18], the magnitude of the current shunted away from the load (referred to as "current loss") varies with the load inductance. The mechanisms and magnitude of this loss change with current density, which is a consideration when determining the radial location of the current adder.

The simulation analysis proceeds from outer to inner radii. The power flow in Z's four radial outer feeds and adder section is detailed in Sec. III. The power diverted from the load in this region is a result of uninsulated free current flows. The plasma desorbed in the adder section contributes additional current to this loss. The magnetic field in the load region would result in insulated sheath currents if not for shear instabilities accompanied by a Hallfield-related transport described in Sec. IV.

\section{SIMULATION MODEL}

In the $Z$ machine, 36 pulsed-power modules are connected in parallel to four radial magnetically insulated transmission lines (MITLs), labeled "outer MITLs" in Fig. 1. Currents from the outer MITLs are combined in a double post-hole convolute architecture to deliver up to $26 \mathrm{MA}$ in $100 \mathrm{~ns}$ to the load. The post-hole convolute, also used in the PTS and MIG generator, is located either 7.5 or $15.5 \mathrm{~cm}$ from the load and so is not well resolved in Fig. 1. Downstream from the convolute is a single inner MITL the transports the full current to the load. These three sections of hardware are located within the dashed lines in Fig. 1 and are tailored for specific loads on $Z$.

The $Z$ machine fields two convolute configurations, one with 12 posts equally spaced on a 15 -cm-diameter $(\varnothing 15 \mathrm{~cm})$ circle [18], illustrated in Fig. 2, and one with a $31-\mathrm{cm}$-diameter $(\varnothing 31 \mathrm{~cm})$ circle. Many inner MITL geometries are fielded with different AK gap spacings and axial extents, and may be characterized by their total inductances. The inner electrodes are subject to the highest current densities and are, therefore, most susceptible to surface plasma formation.

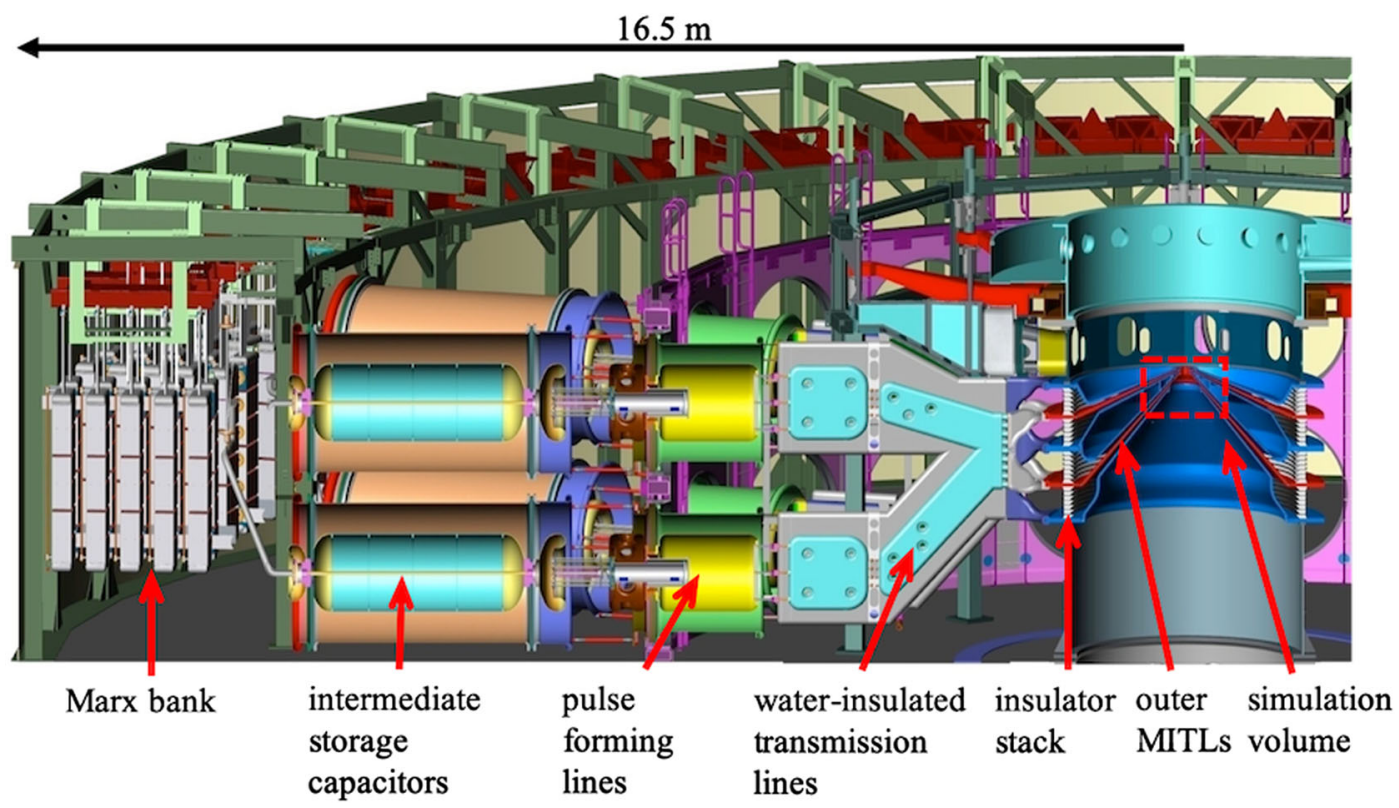

FIG. 1. Cross section of Sandia's $Z$ machine [3,4]. The electrical characteristics of the components shown are represented in PIC simulations by transmission-line-circuit model elements. The 3D simulation volume is outlined by the dashed red lines. The arrows indicating the insulator stack and outer MITLs point to the location of anode B-dot probes for those elements. 


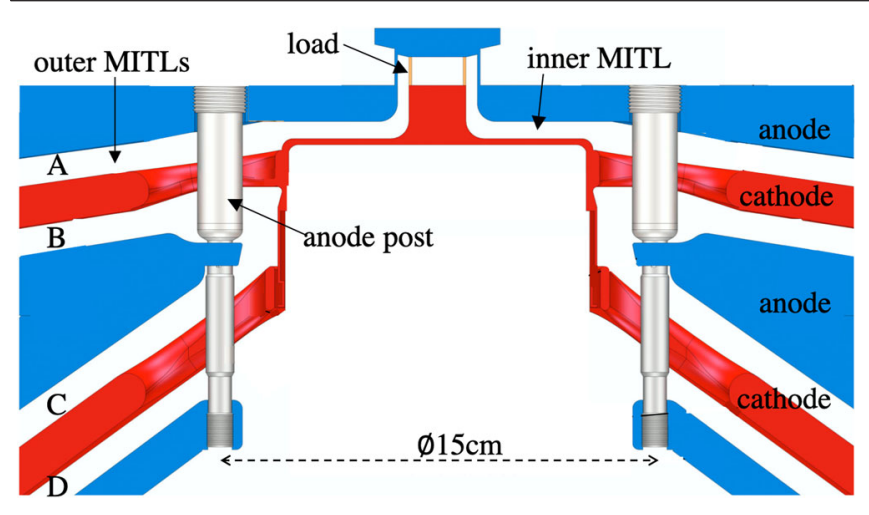

FIG. 2. A cross section of the $Z$ machine outer MITLs, $\varnothing 15 \mathrm{~cm}$ double post-hole convolute (current adder), and inner MITL [19].

To study the generation and evolution of these electrode plasmas, simulations of various $Z$ machine convolute and inner MITL configurations are conducted using the fullyrelativistic electromagnetic particle-in-cell code CHICAGO [14,20-22]. Simulations of both convolutes are conducted in $3 \mathrm{D}$ cylindrical coordinates $(r, \theta, z)$, spanning $15^{\circ}$ azimuthally with symmetry boundaries at $\theta=0^{\circ}$ and $15^{\circ}$ and $100 \mu \mathrm{m}$ maximum resolution. The simulation geometries, shown in Fig. 3, include the final $8 \mathrm{~cm}$ of the outer MITL, the double post-hole convolute, the inner MITL, and load region.

Current distributions derived from the 3D simulations are injected through the boundaries in 2D simulations of additional inner MITL configurations, in the azimuthally symmetric region interior to the convolute. These 2D simulations enable faster comparisons of the different geometries at higher resolution $(\Delta r, z=25-50 \mu \mathrm{m})$ while using the same field solution and particle treatments as in $3 \mathrm{D}$.

The circuit model components of Ref. [9] provide a realistic $Z$ driving pulse. Four voltage pulses are supplied to a BERTHA [23] circuit originating at the water convolute, just outside the insulator stack. In 3D models, the circuit is connected at four grid boundaries to ideal radial or axial feed sections to minimize longitudinal electric fields that are not modeled in the 1D circuits. When needed to accurately model shot data, an imploding-load impedance is modeled using a second circuit [24].

The simulations use a magnetic implicit electromagnetic field solver to advance the particles and fields, which relaxes the time step constraint due to cyclotron motion. Reference [12] tabulates allowable values of $\omega_{c} \Delta t$ to be between 3 and 30 depending in the cyclotron radius and strength of the magnetic field gradient. The simulations presented here use $\omega_{c} \Delta t \leq 2$. An energy-conserving cloud-in-cell model is used to minimize electromagnetic fluctuations from individual macroparticles [25] and a time-biasing algorithm [26] is used to control the growth of electromagnetic fluctuations on the grid. The adaptive particle management is used to limit the number of particles
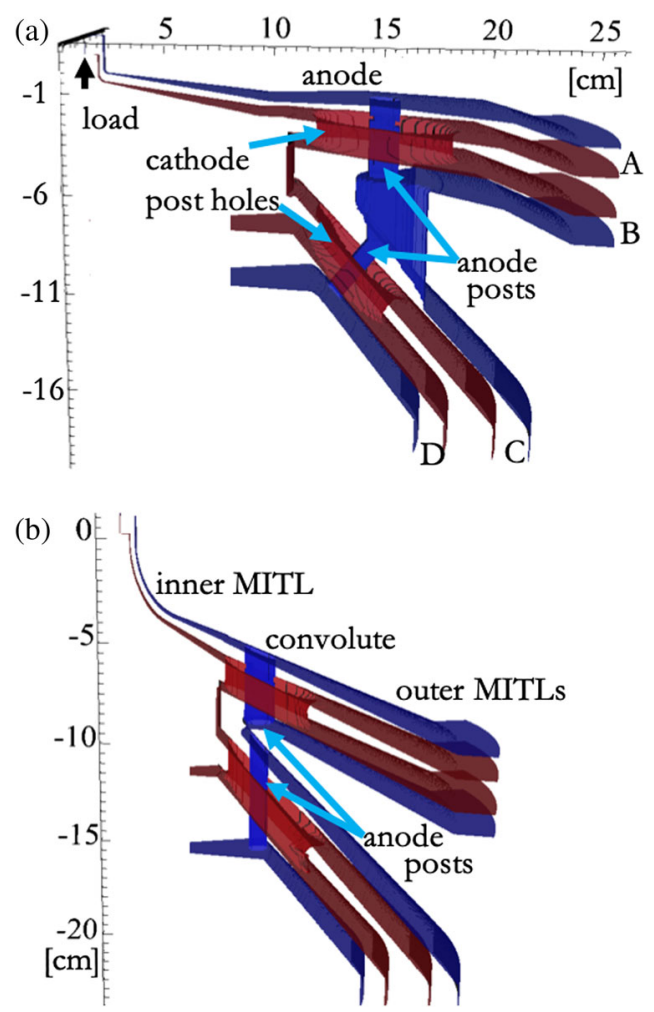

FIG. 3. Illustration of the outer MITLs, double post-hole convolute, and inner MITL fielded for (a) a $\varnothing 31 \mathrm{~cm}$ convolute and (b) a $\varnothing 15 \mathrm{~cm}$ convolute. A pulse-generating circuit is attached at the four outer MITL feeds labeled A, B, C, and D.

per species per cell to 150 , when the charge, energy, and momentum distributions may all be preserved.

The electrode surfaces are continuously updated for local temperature increases from Joule heating and particle energy deposition. The Joule heating model derives from [27]

$$
T_{J}(t)=\frac{1}{c_{v}} \int_{0}^{t} \frac{j^{2}(t)}{\sigma} d t
$$

where $\sigma$ and $c_{v}$ are the conductivity and specific heat (per unit volume) of the electrode material (assumed constant) and the lineal current density is determined from the curl of the magnetic field: $j(t)=\partial H(x, t) / \partial x$, where $H=B / \mu_{0}$ and $x$ is the coordinate normal to the conductor for the surface cell being evaluated. Assuming a linearly rising $j(t)$ and that magnetic field diffusion is the dominant contributor over heat conduction, the temperature increase on a cellby-cell basis is

$$
\Delta T_{J}(t) \approx \frac{\vartheta \mu_{0} H^{2}(t)}{2 c_{v}},
$$

which is Eq. (5.2)-(30) from Ref. [27]. The surface energy factor $\vartheta=1.273$ (from Table 5.2-II of Ref. [27]) 
and $c_{v}=3.9 \mathrm{~J} / \mathrm{cm}^{3} \mathrm{~K}$ for stainless steel $[28,29]$. As noted in Ref. [27], the assumption of constant $\sigma$ underestimates the temperature rise but is valid for small $\delta T / T$.

Particle energy deposition $(d E / d s)$ is calculated using the Bethe-Bloch equations for electron and ion energy loss [30]. $d s$ is the path length in a grid cell, $d s=d x / \cos \theta$, where $d x$ is the cell depth and $\theta$ is the particle angle of incidence. The temperature increase $\left[T_{d}(t)\right]$ per macroparticle is then calculated as

$$
\Delta T_{d}=\frac{d E}{d s} \frac{q}{e c_{v} A},
$$

where $q$ is the macroparticle charge, $e$ is the electron charge, and $A$ is the cell's surface area.

Particle emission is modeled as both field and thermal emission of charged particles from the electrode surfaces and thermal desorption of neutral plasma. Electrons are emitted from the cathode after the local electric field stress exceeds the tolerance of the conductor [17,31]. The emission threshold depends on the material and its preparation, with typical values ranging from 150 to $280 \mathrm{kV} / \mathrm{cm}$ as determined when the anode current exceeds the cathode boundary current $[15,16]$. A previously tested value of $240 \mathrm{kV} / \mathrm{cm}$ [11] is used here. Once this threshold is exceeded, electron emission is space-charge-limited. Protons are emitted from the anode after the local surface temperature increases by $400 \mathrm{~K}$ [32]. The emission rate for these processes is governed by the space-charge-limited (SCL) current, the nonrelativistic version of which is [17]:

$$
j_{\mathrm{CL}}=2.34 \times 10^{-6} V_{0}^{3 / 2} / d^{2}\left[\mathrm{~A} / \mathrm{cm}^{2}\right],
$$

where $V_{0}$ is the voltage drop in $\mathrm{V}$ and $d$ is the AK gap width in $\mathrm{cm}$.

Both the field-stress breakdown of the cathode and the thermal breakdown of both electrodes lead to the creation of local surface plasmas. To model plasma formation, a neutral atom or molecule is desorbed from an electrode surface and immediately fragmented to its atomic constituents and ionized, as in Refs. $[33,34]$. Previous measurements at Sandia have identified hydrogen, oxygen, and carbon as the primary electrode surface contaminants in the SABRE accelerator [35] and as negative ions in prototypes of the Particle Beam Fusion Accelerator [36]. More recent spectral measurements on the $Z$ machine identify hydrogen as the primary plasma constituent [18]. While carbon was included in models in Ref. [34], only hydrogen and oxygen are used here to reduce the number of tracked species. Higher states and excitation are also not modeled for this reason.

Preliminary simulations included avalanche ionization $\left[e+\mathrm{H}(\mathrm{O}) \rightarrow 2 e+\mathrm{H}^{+}\left(\mathrm{O}^{+}\right)\right]$and electron attachment $\left[e+\mathrm{H}(\mathrm{O}) \rightarrow \mathrm{H}^{-}\left(\mathrm{O}^{-}\right)\right]$, with cross sections that peak near $100 \mathrm{eV}$ and $10 \mathrm{eV}$, respectively. Because the sheath electron energies may exceed an $\mathrm{MeV}$, these simulations only generated partially $(<10 \%)$ ionized plasmas and negligible negative ions. Test simulations indicate that adequate models for these processes require seed populations of low-energy electrons and cell sizes less than $10 \mu \mathrm{m}$. A more refined analysis including avalanche ionization, photoionization, attachment, and the expected plasma ionization fraction will be the subject of a future investigation. For the simulations presented here, thermal ionization [37] is assumed.

The $\mathrm{H}_{2} \mathrm{O}$ molecule and its constituents are tracked as kinetic species. The molecule is fragmented and subsequently ionized according to: $\mathrm{H}_{2} \mathrm{O} \rightarrow 2 \mathrm{H}^{+}+\mathrm{O}^{+}+3 e$. The Arrhenius equation calculates the thermal desorption rate as a function of the surface density of adsorbed contaminant particles $[n(t)]$ :

$$
\frac{d n(t)}{d t}=-\nu_{t h} n(t) e^{-E^{\prime}(n) /\left[k_{B} T(t)\right]}
$$

where $\nu_{\text {th }} \sim 10^{13} \mathrm{~s}^{-1}, E^{\prime}(n)$ is the effective binding energy, and $T$ is the local surface temperature [35,38]. The surface density is measured in monolayers, $n=f n_{\mathrm{ML}}$, where $n_{\mathrm{ML}} \equiv 10^{15}$ particles $/ \mathrm{cm}^{2}$ and $f$ is a surface roughness factor [39] ( $f \sim 8$ for average machining of stainless steel [40]). The Temkin isotherm model has been shown to capture the surface-density dependence of $E^{\prime}(n)$ seen in molecular dynamics simulations of $\mathrm{Fe}_{2} \mathrm{O}_{3}$ lattices [41]:

$$
E^{\prime}(n)=E_{d}\left(1-\alpha \frac{n(t)}{f n_{\mathrm{ML}}}\right)[\mathrm{eV}],
$$

where $E_{d}$ is the binding energy at infinitesimal coverage, $\alpha$ is determined experimentally. The values $E_{d}=1 \mathrm{eV}$ and $\alpha=0.17$ are used here. The initial contaminant inventory on the electrode surfaces, $n(0)$, is $8.3 \mathrm{ML}$.

The sensitivity to the parameters used in Eqs. (1) and (4) were tested in simulations in which $d n(t) / d t$ was varied by a factor of 6. In this range, there was no change in the plasma transport presented in Sec. IV. However, reducing $d n(t) / d t$ by 2 orders of magnitude virtually eliminates plasma transport.

\section{A. Particle interactions}

The electrons, ions, and neutrals are all governed by kinetic particle algorithms. Thus, the particles are advanced via their individual momenta, collision frequencies, and interaction cross sections, with no assumed distributions. The ionization of neutral particles is expected to occur rapidly due to high electric field stresses and collisions with sheath electrons. The electron-ion pairs created in ionization events are added to their respective kinetic species. Ion-neutral collisions are also handled in binary fashion assuming a hard-sphere collision model.

Charged particle collisions use a binary Coulomb collision model derived from Ref. [42], in which Nanbu's 
theory of the cumulative property of Coulomb collisions is applied. Individual particles located within the same grid cell are paired and a Monte-Carlo algorithm determines the effective cumulative Coulomb collision scattering angle for the pair. The correct local velocity distribution is sampled over successive time steps.

\section{B. Simulation resolution}

A reliable method for determining the minimum resolution required for MITL flow is to compare simulation results to the transmission line theories of Creedon [43] and Mendel and Rosenthal [44]. For laminar sheath flow, both theories relate the voltage drop across the transmission line $\left(V_{0}\right)$ to the anode current $\left(I_{a}\right)$, the cathode boundary current $\left(I_{c}\right)$, and vacuum impedance $\left(Z_{0}\right)$. The relation derived from Ref. [43] is

$$
\begin{aligned}
V_{0}= & 0.511\left(\gamma_{m}-1+\left(\gamma_{m}^{2}-1\right)^{1 / 2}\left[\frac{I_{c} Z_{0}}{I_{\alpha} Z_{\alpha}}\right.\right. \\
& \left.\left.-\ln \left[\gamma_{m}+\left(\gamma_{m}^{2}-1\right)^{1 / 2}\right]\right]\right)[V] .
\end{aligned}
$$

where $Z_{\alpha}=60 \Omega, I_{\alpha}=(2 \pi)\left(\mu_{0} c\right)\left(m_{e} c^{2}\right)(e) \approx 8500 \mathrm{~A}$, and $\gamma_{m}=I_{a} / I_{c}$ is also the relativistic factor at the extent of the sheath. This results in the MITL operating at an impedance $\left(Z_{\mathrm{op}}\right)$ that is less than $Z_{0}$.

Agreement between $\int \mathbf{E} \cdot d \mathbf{l}$ and $V_{0}$ from Eq. (5) for MITL flow may be regarded as a test of simulation accuracy, even for systems with a dynamic $Z_{\text {op }}$ [15]. We determined the impact of spatial resolution on simulation accuracy using a $2 \mathrm{D}$ coaxial MITL at $40 \mathrm{kA}$. Using the difference between $\int \mathbf{E} \cdot d \mathbf{l}$ and Eq. (5) as the metric, the simulations converged to the accurate solution as the spatial resolution increased from 0.5 to $0.25 \mathrm{~mm}$. Increasing the temporal resolution alone had no effect.

The magnitude of the electron current liberated from the cathode in SCL emission is defined by Eq. (3), after which the electrons are accelerated by $E_{r}$ in the grid cell adjacent to the conductor. As the resolution decreases, $\Delta E_{r}$ in each cell increases and the electron begins sampling a local field value that is too large. The electrons are transported to larger radius than they would with proper resolution. The sheath thus becomes broader, $Z_{\text {op }}$ is reduced, and the anode and cathode boundary currents are increased.

The electron cyclotron radius need not be resolved for accurate MITL flow, however $\omega_{c} \Delta t$ should be resolved in PIC simulations using the Boris push [45]. This requirement is relaxed in CHICAGO when the magnetic-implicit algorithm is used. Any schemes that relax the $\omega_{c} \Delta t$ and $\omega_{p} \Delta t$ constraints do not mitigate the grid resolution requirement for MITL simulations.

Resolution scans of a coaxial MITL demonstrate that this artificial sheath broadening will appear as a smaller effective $\mathrm{AK}$ gap, reducing $Z_{\mathrm{op}}$, and will exhibit

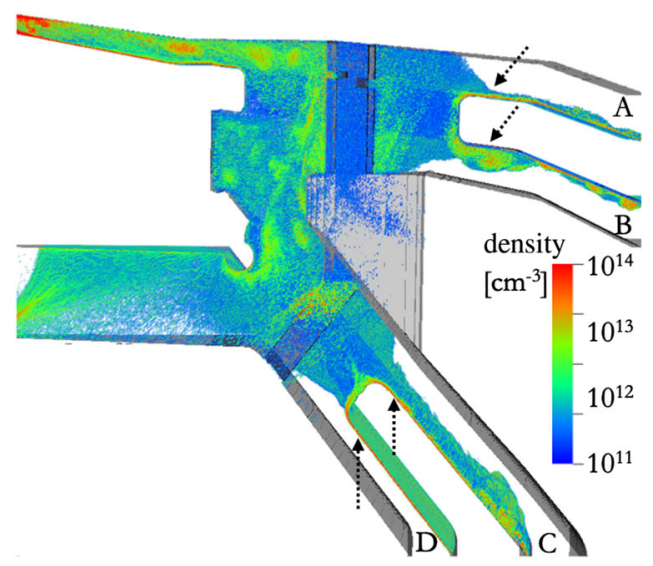

FIG. 4. Electron density contours in the $\varnothing 31 \mathrm{~cm}$ convolute [Fig. 3(a)] during the pulse rise. Arrows mark the locations in each outer MITL where Eq. (5) is compared to the simulation voltage in Fig. 5.

noise-induced turbulent radial motion. The simulation will thus appear to predict flow instabilities and current shunting across the gap where none actually exist.

The test for agreement between $\int \mathbf{E} \cdot d \mathbf{l}$ and Eq. (5) is conducted in each outer MITL for every 3D simulation presented here. $I_{a}, I_{c}$, and $V_{0}$ are recorded at the locations marked by arrows in Fig. 4, which also shows the electron density in the Fig. 3(a) geometry during the pulse rise. The simulated and theoretical voltages, shown in Fig. 5, agree after the sheath insulates in each MITL. Later oscillations in Figs. 5(b) and 5(c) are due to vortices in the flow [15,46]. The 3D simulations presented here have $150-\mu \mathrm{m}$ resolution in the outer MITLs, and no change in agreement is noted when the resolution is reduced to $200-\mu \mathrm{m}$.

\section{CURRENT FLOW IN THE OUTER MITL AND CONVOLUTE}

A second test for simulation relevancy is a comparison of measured to simulated currents feeding into the outer MITLs. On $Z$, anode currents in the insulator stack and outer MITLs are measured with B-dot probes arrayed azimuthally at the radial positions marked in Fig. 1 [47]. B-dot probes are located on all four feeds in the stack and on the A and D feeds in the outer MITL. The azimuthallyaveraged anode currents at each radial position are plotted in Fig. 6. Data from two $Z$ shots are shown and are within $2 \%$ of the corresponding simulated currents. These simulated currents are inside the network of 1D transmissionline segments, outside the 3D simulation volume, but are calculated using the grid's backward-traveling voltages and currents.

Inside the simulation 3D grid, the electron density distributions in Fig. 4 show flow in the outer MITLs is magnetically insulated with rolling vortices. Insulated flow ends at the convolute where the fields $\left(B_{\theta}, E_{r, z}\right)$ change sign. The drift path of the free current through the convolute 


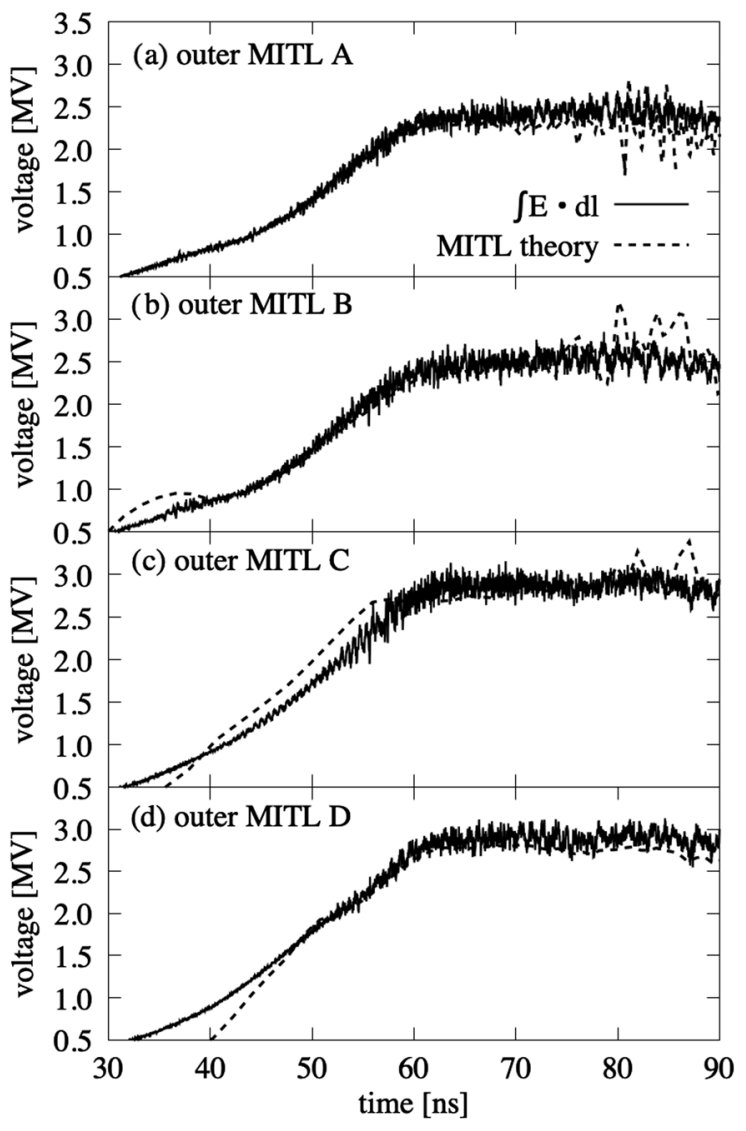

FIG. 5. The voltage drop across the four outer MITL gaps from the simulated electric fields compared to pressure-balance-theory of Eq. (5).

is illustrated by the $\mathbf{E} \times \mathbf{B}$ vectors in Fig. 7. Both Figs. 4 and 7 indicate that particles within the convolutes flow toward and along the anode posts to the top anode plate. Some of the vectors shown are in the azimuthal direction or toward the electrodes. The sheath entering the inner MITL is not well insulated and deposits energy in the cathode and anode surfaces, increasing the surface temperatures, in addition to Joule heating.

To determine the impact of particle energy deposition in the convolute, we first examine the impact of Joule heating alone. Using the current pulse delivered to a load without losses [Fig. 8(a)], the surface temperature is calculated using Eq. (1) for a range of radii within the inner MITL [Fig. 8(b)]. The temperature threshold for neutral desorption is rapidly exceeded at small radius, with desorption at $r=1 \mathrm{~cm}$ by $50 \mathrm{~ns}$. Joule heating alone initiates desorption $(700 \mathrm{~K})$ to $r \leq 9.1 \mathrm{~cm}$, which includes the entire convolute region for the $\varnothing 15 \mathrm{~cm}$ version.

The rise in surface temperature for each outer MITL is plotted in Fig. 8(c) using lossless feed currents. Because the current density in the outer MITLs reaches only $40-100 \mathrm{kA} / \mathrm{cm}^{2}$, power loss in this region occurs only through uninsulated free current flows. Figures 8(b) and 8(c) show thermally desorbed anode plasmas would

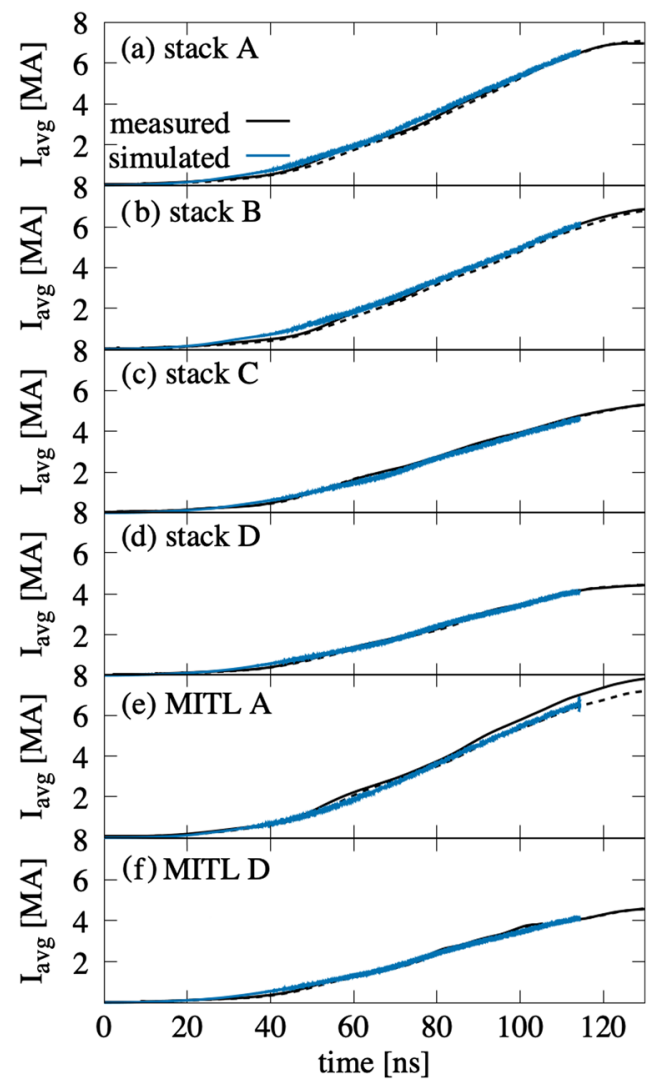

FIG. 6. The measured and simulated currents in the stack and outer MITLs for the Fig. 3(a) hardware.

not form in the $\varnothing 31 \mathrm{~cm}$ convolute, where current densities downstream from the anode posts are $300-400 \mathrm{kA} / \mathrm{cm}^{2}$, if not for particle energy deposition. Electrons impact the anode posts, center, and top plate, depositing energy in a similar pattern in each convolute, as shown in Fig. 9. As a result, the $\varnothing 31 \mathrm{~cm}$ convolute has localized plasma formation on the anode posts and plates. As reported in Ref. [11], the $\varnothing 15 \mathrm{~cm}$ convolute will have plasma covering the inner MITL including the downstream sides of the posts.

The current lost to the convolute surfaces resulting from particle energy deposition is amplified as plasma forms. To quantify this current, the combined losses $\left(I_{\text {loss }}\right)$ in the outer MITLs and convolute are plotted in Fig. 10 for four configurations fielded on $Z$. The first trace in Fig. 10 comes from the Fig. 3(a) power-flow study. The second trace uses the same convolute but an alternate inner MITL of similar inductance (within 1\%). The third trace comes from a $\varnothing 15 \mathrm{~cm}$-convolute. The fourth uses the $\varnothing 15 \mathrm{~cm}$ convolute with the MagLIF inner MITL [24,48,49], which is extended in the axial direction as shown in Fig. 3(b).

For the first $27 \mathrm{~ns}, I_{\text {loss }}$ is the same for all four geometries. The transmission lines operate in vacuum for the first $12 \mathrm{~ns}$ of the pulse during which $I_{\text {loss }}=0$. This is followed by electron emission from the cathodes. In the outer MITLs, this free electron current insulates by $30 \mathrm{~ns}$, 


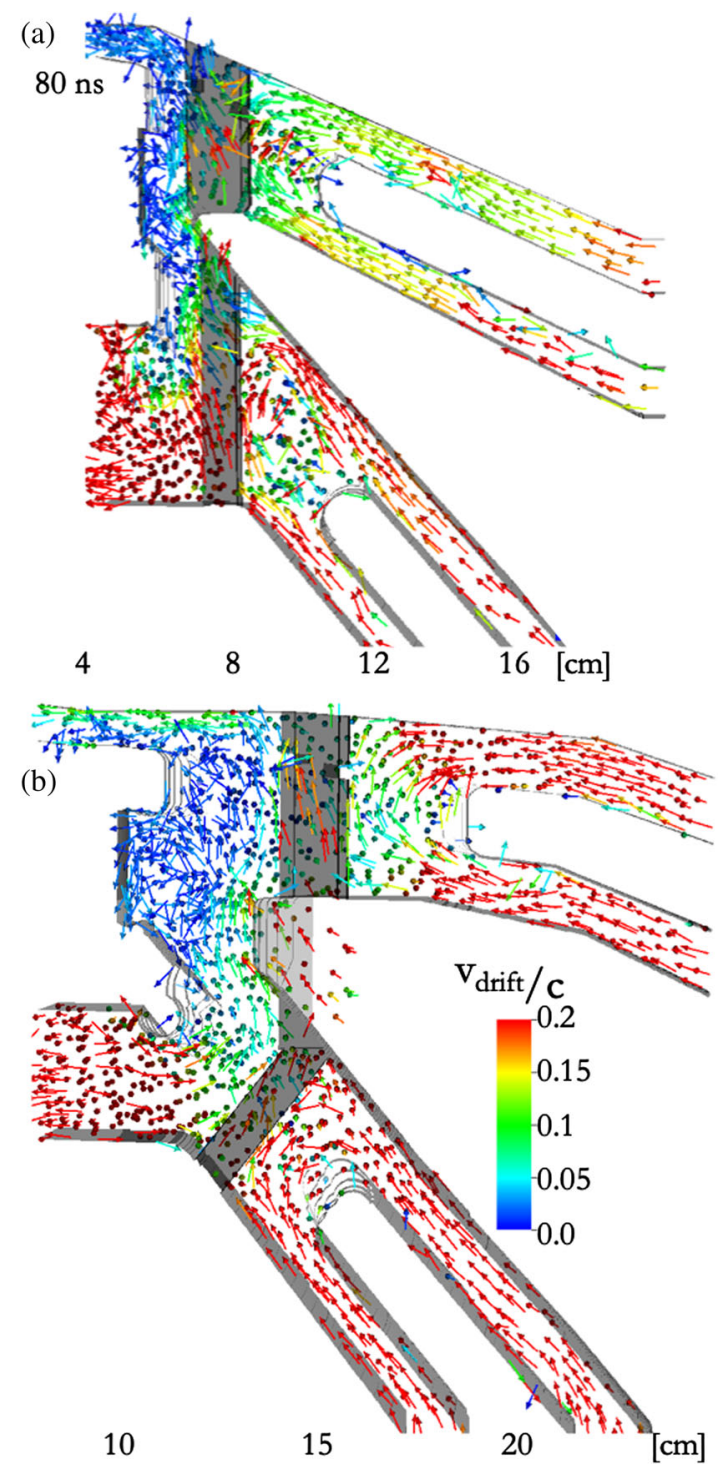

FIG. 7. $\quad \mathbf{v}_{\text {drift }}=\mathbf{E} \times \mathbf{B} / B^{2}$ in (a) the $\varnothing 15 \mathrm{~cm}$ and (b) the $\varnothing 31 \mathrm{~cm}$ convolutes at $80 \mathrm{~ns}$.

after which $\sim 100 \mathrm{kA}$ still crosses the AK gap. This current loss is due to the impedance transitions near the cathode post holes where the flow is no longer laminar. MagLIF uses an applied $B_{z}$ and this delays insulation until $B_{\theta} \gg B_{z}$ at $\simeq 40 \mathrm{~ns}$.

After outer MITL insulation, $I_{\text {loss }}$ is greater in the $\varnothing 31 \mathrm{~cm}$ convolute, with electrons impacting a larger area on the center and top anodes, as seen in Figs. 7 and 9. The drop in $I_{\text {loss }}$ between 40 and $50 \mathrm{~ns}$ in Fig. 10 corresponds to a drop in the particle flux entering the convolute from each outer MITL after electrons are recaptured in the cathode [15] but prior to plasma formation.

Surface plasmas form in the $\varnothing 15 \mathrm{~cm}$ convolutes by 70 ns. The average local plasma density, calculated from the total particles in the convolute, is represented by dashed lines in Fig. 10. For both $\varnothing 15 \mathrm{~cm}$ configurations, $I_{\text {loss }}$ increases with plasma density. The ion density map in

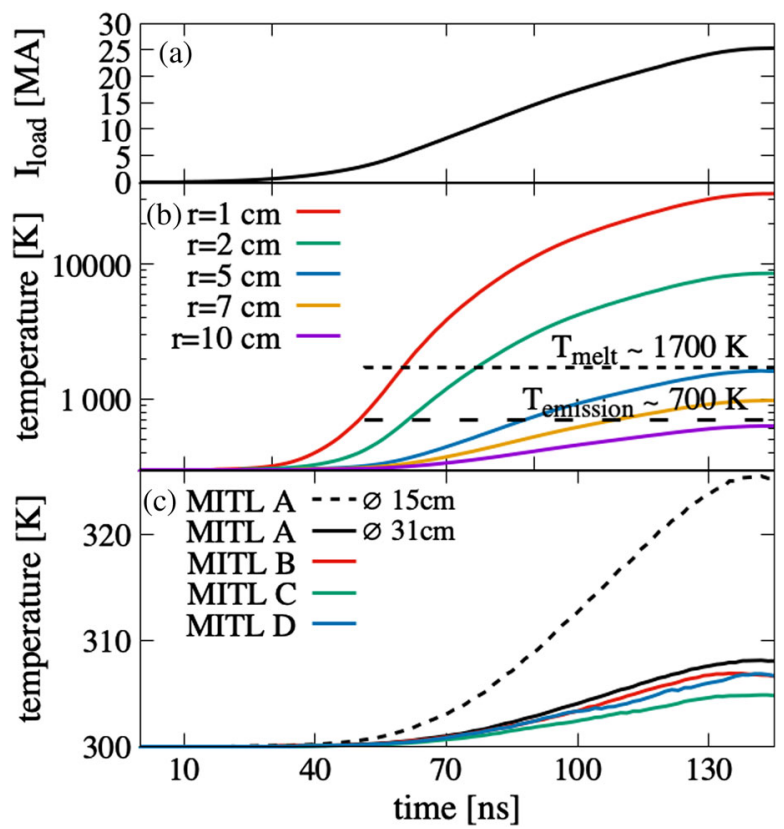

FIG. 8. (a) The current pulse delivered to the load without losses. (b) Electrode surface temperatures at various radii in the inner MITL calculated using Eq. (1) and the pulse in (a). (c) Electrode surface temperatures calculated at the inner radius of each outer MITL as labeled in Fig. 3. The $\varnothing 31 \mathrm{~cm}$ convolute is represented by solid lines. The $\varnothing 15 \mathrm{~cm}$ is represented by a dashed line.

Fig. 11(a) illustrates the initial plasma evolution. As expected, plasma forms on the Joule-heated entrance to the inner MITL and surfaces that are bombarded by electrons. The surface plasmas contribute charged particles to the existing free particle currents where currents are not magnetically insulated. We define the thermal energy of a species as the spread in the species' momenta about the mean in a cell. The electron and ion densities and thermal energies range from $10^{16} \mathrm{~cm}^{-3}$ and $100 \mathrm{eV}$ on the electrode surfaces to $10^{13} \mathrm{~cm}^{-3}$ and $100 \mathrm{keV}$ in the gap.

The high magnetic fields in the convolute (25-30 T with $\omega_{c e} / \nu_{e i}>10^{4}$, where $\nu_{e i}$ is the electron-ion collision frequency) would be expected to impede standard transport (uninsulated charged-particle flows) across the AK gap if not for the magnetic nulls, impedance transitions, and resulting $\mathbf{v}_{\text {drift }}$ map in Fig. 7). (The loss near the magnetic nulls, first reported in Ref. [50] is illustrated by the particle energy deposition on the lower anode posts in Fig. 9. Little loss is observed in the magnetic null in the upper post hole.) In addition, the density and velocity gradients result in velocity shear instabilities on both electrode surfaces from which filaments expand into the gap, as shown in Fig. 11(b). This effect is delayed for the $\varnothing 31 \mathrm{~cm}$ convolute.

The instability growth in magnetically insulated electron sheaths has been analyzed previously for idealized MITLs. Reference [51] determined that the growth rate of transverse-magnetic waves scales with the shear in the drift 


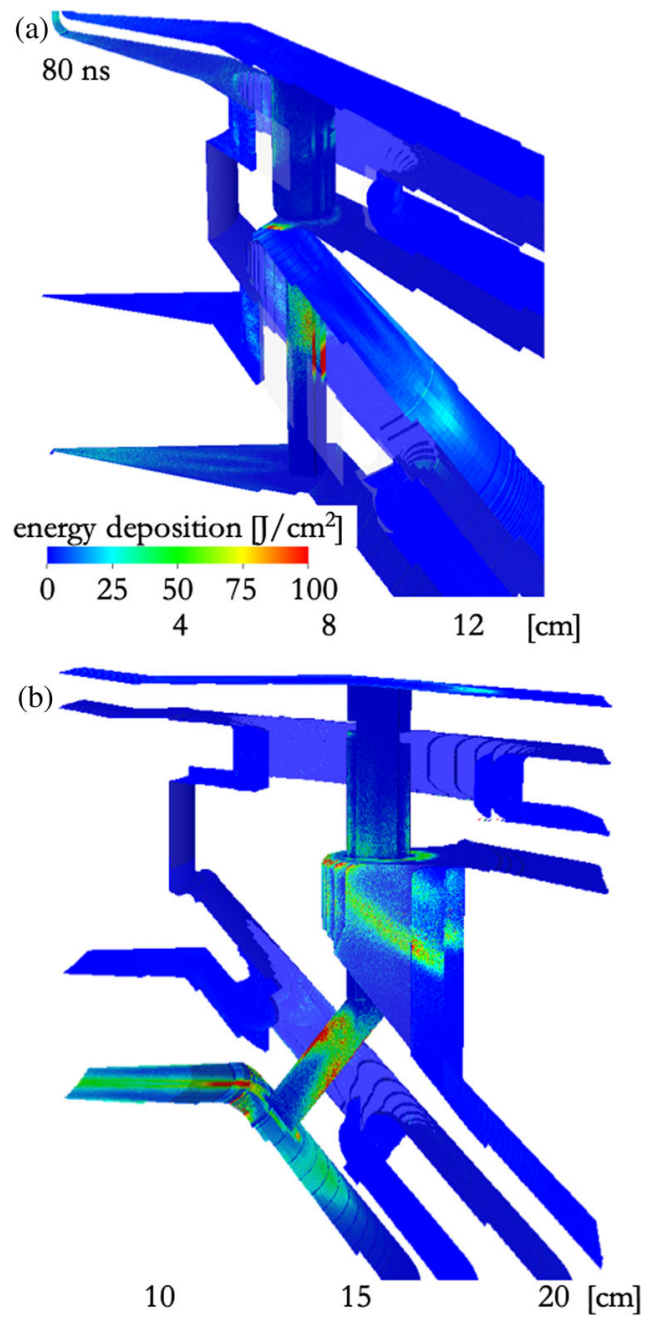

FIG. 9. Cumulative particle energy deposition in the (a) $\varnothing 15 \mathrm{~cm}$ and (b) $\varnothing 31 \mathrm{~cm}$ convolutes at $80 \mathrm{~ns}$.

velocity of electrons near the sheath/vacuum interface. Reference [52] determined that including anode resistivity extends the range of unstable frequencies. Reference [53] determined that a static resistive plasma layer between

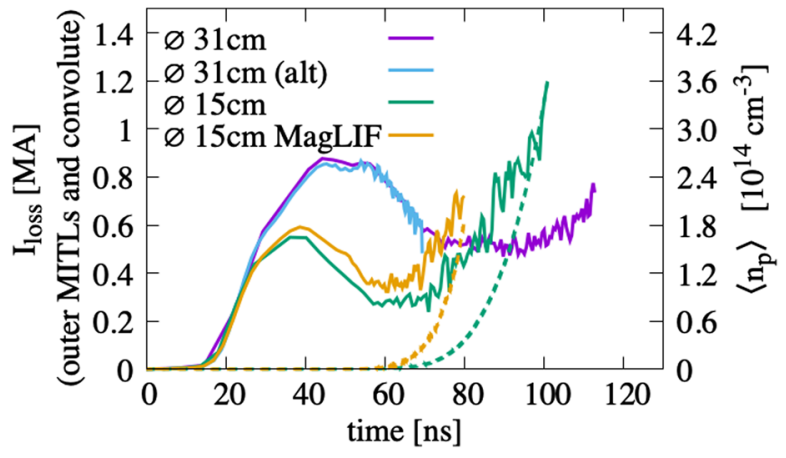

FIG. 10. The current loss in the outer MITLs and convolute for two $\varnothing 31 \mathrm{~cm}$ and two $\varnothing 15 \mathrm{~cm}$ convolute configurations. The two $\varnothing 31 \mathrm{~cm}$ examples have similar load inductances while the two $\varnothing 15 \mathrm{~cm}$ differ by $80 \%$. Dashed lines represent the mean proton densities in the $\varnothing 15 \mathrm{~cm}$ convolutes, matched by color.
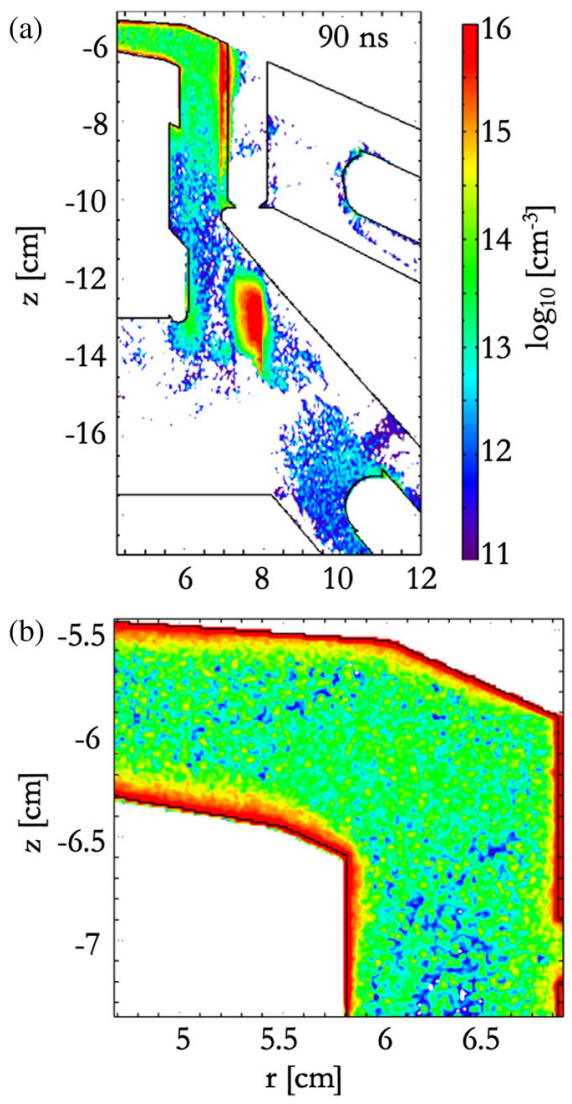

FIG. 11. Ion density contours in the $\varnothing 15 \mathrm{~cm}$ convolute at 90 ns shown for (a) the anode posts' surfaces and (b) a magnified view near the inner MITL. The densities are plotted on a $\log$ scale from $10^{11}-10^{16} \mathrm{~cm}^{-3}$.

the cathode and sheath modifies the modes supported by the electron sheath and introduces a broad spectrum of low-frequency modes which can couple to perturbations in the sheath.

More recently, Refs. [54] and [13] modeled more realistic MITLs with dynamic cathode plasmas and found that the combination of plasma and sheath instabilities enhanced the plasma expansion into the MITL gap. The instabilities modeled in Ref. [13] are consistent with the resistive-plasma model and relevant to the plasma-sheath interactions modeled here. In these integrated simulations of plasma formation and power flow, plasma is continuously swept downstream due to the ponderomotive force. Although the processes are similar, the presence of these plasmas complicates the sheath interactions.

\section{POWER DELIVERY IN THE INNER MITL}

The currents combined in the $Z$-machine convolute are fed into the inner MITL, a radial transmission line with a load-dependent design. Two examples of inner MITL geometries are illustrated in Fig. 3. While the rate at which the electrode surfaces reach emission threshold varies with geometry, typically the electric field stresses generate 
cathode plasmas $15 \mathrm{~ns}$ after the pulse arrives in this section of $Z$, when the current is near $70 \mathrm{kA}$. The magnetic fields near the load exceed $100 \mathrm{~T}$ by $50 \mathrm{~ns}$ which impedes charged-particle flows across the AK gap.

At these magnetic pressures without instabilities, the plasmas are confined near the electrode surfaces. However, the free current flowing from outer to inner radii, with an energy gradient of $1-100 \mathrm{keV}$ from the surface into the gap, provides a driver for velocity shear and resistive-plasma instabilities. Figure 12 shows the ion density distribution in the inner MITL, including a magnified view of the electrode surfaces, from the hardware in Fig. 3(a). For this simulation, the electrode at the inner radius modeled a static load that did not emit particles. This results in an artificially low plasma density near the load. For the rest of the inner MITL, the density increases with decreasing radius, as expected from surface heating. The density distribution from the electrode surfaces into the gap exhibits plumes from the resistive plasma and other shear instabilities. The calculated transport is qualitatively similar to that described in Ref. [13] and is likely the result of the same resistive plasma-sheath instability. The nonlinear state of the instability leads to uncovered electron and ion charge which drives plasma across the magnetic field. This highly turbulent process continues into the gap yielding large electric field fluctuations of submillimeter scale.

2D simulations explore how changes in the inductances of fielded inner MITLs (length, radial convergence, and AK gap) impact the density distributions and power delivered to the load. These variations in geometry, such as illustrated in
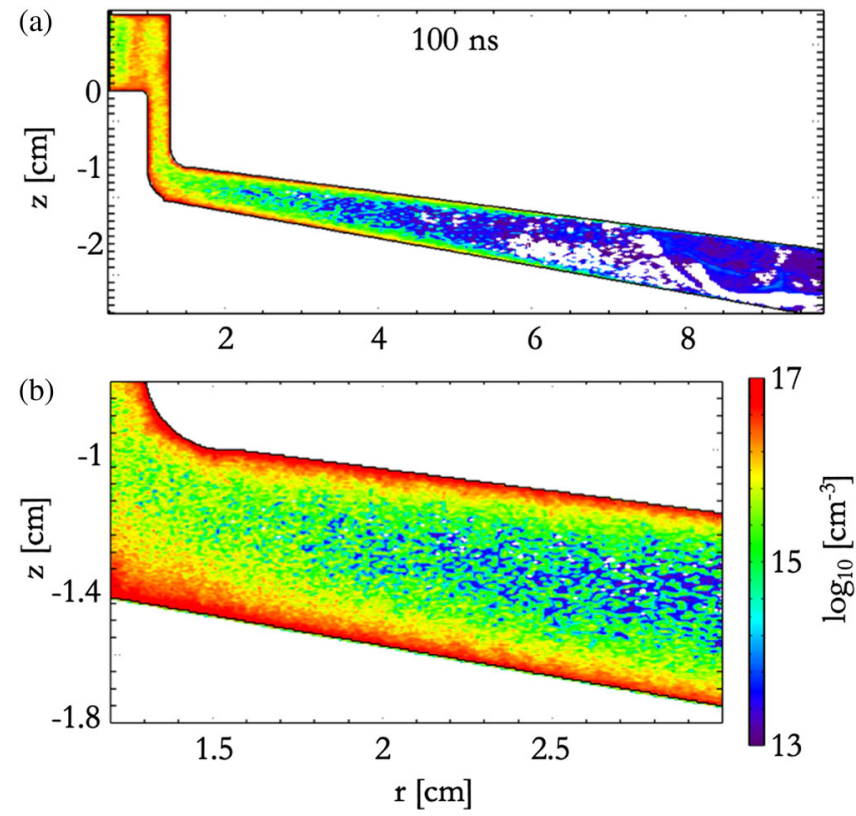

FIG. 12. (a) Ion density contours in the inner MITL at $100 \mathrm{~ns}$ with (b) a magnified view. This is the inner MITL from the geometry in Fig. 3(a). The densities are plotted on a log scale from $10^{13}-10^{17} \mathrm{~cm}^{-3}$.
Fig. 3, do delay the transport of a $10^{15}-\mathrm{cm}^{-3}$-density plasma into the gap, but in all cases transport is volumetric via sub-millimeter-sized filaments. The simulations use $\Delta r, z=25-50 \mu \mathrm{m}$ and $\Delta t=1.5 \times 10^{-5} \mathrm{~ns}$ to resolve the particle and field structures, with $\Delta r, z<3$ skin depths in the dense-plasma regions.

The plasma transport leads to a volumetric current loss that increases with the density in the gap. An example of the current delivered to the load $\left(I_{\text {load }}\right)$ modified by losses is provided in Fig. 13 for the geometry in Fig. 3(a). The peak $I_{\text {load }}$ is 3 MA less than the lossless current in Fig. 8(a).

Figure 13 also compares the simulated $I_{\text {load }}$ to load current inferred from a velocimetry diagnostic [55]. Two load variants are plotted; one is the simulation in Fig. 12 and one has a similar shape but with $5 \%$ lower inductance. The dip in the measured current at 90 ns relative to simulation is under investigation.

Inductance is used in Fig. 14 as the label distinguishing the various inner MITL configurations. It is relevant because it is a function of the geometric structures that increase plasma transport and current loss across the AK gap. Namely, inductance increases with increasing transmission line length and decreasing radius for coaxial lines. The total length is significant because the current loss is volumetric. The decreasing radius is significant because it increases current density in the electrodes and, thus, hastens heating and desorption. The relationship of inductance to current loss is approximate and notable exceptions are configurations that use an applied $\mathbf{B}$ field and radial transmission lines with increasing gap width.

The inner MITL configurations fielded on $Z$ aim for low inductance, typically less than $7 \mathrm{nH}$ after the convolute. A comparison of the current lost within the inner MITL $\left(I_{\text {loss }}\right)$ for simulations of four load inductances is shown in Fig. 14. These simulations show that $I_{\text {loss }}$ decreases with decreased load inductance.

The current flow parallel and perpendicular to the electrodes in the inner MITL is a turbulent process complicated by the fields generated through submillimeterscale charge separations described above. The particle

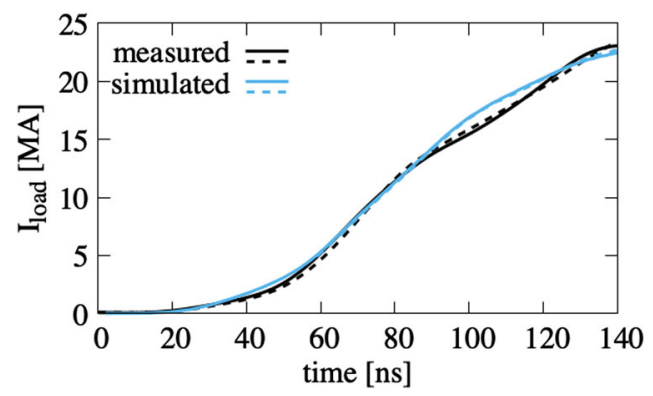

FIG. 13. The simulated and measured currents delivered to the load $\left(I_{\text {load }}\right)$ for the geometry in Fig. 3(a) (solid) and a variant with similar inductance (dashed). The measurement uncertainty is $\pm 5 \%$. 


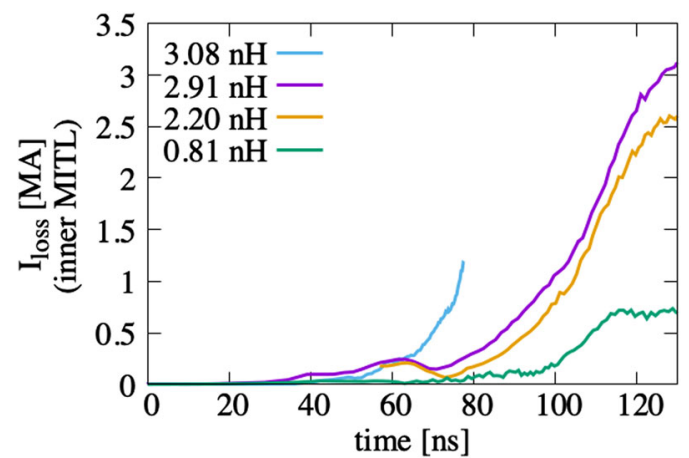

FIG. 14. The net current shunted across the AK gap in the inner MITL $\left(I_{\text {loss }}\right)$ from simulations of four inner MITL geometries identified by their load $(r \leq 1.3 \mathrm{~cm})$ inductances.

densities at the electrode surfaces exceed $10^{17} \mathrm{~cm}^{-3}$ while the bulk of the plasma responsible for cross-gap current has density between $10^{14}-10^{16} \mathrm{~cm}^{-3}$, as shown in Fig. 12 . We draw on a fluid description of conductivity to form an idealized picture of this flow, while noting that the fields and currents are only calculated self-consistently in a kinetic treatment.

The fluid electron equation of motion is

$$
\begin{aligned}
m_{e} n_{e} \frac{d \mathbf{v}_{e}}{d t}= & -e n_{e}\left(\mathbf{E}+\mathbf{v}_{e} \times \mathbf{B}\right)-\nabla p_{e} \\
& +\sum_{\alpha} \nu_{e \alpha} m_{e} n_{e}\left(\mathbf{v}_{e}-\mathbf{v}_{\alpha}\right),
\end{aligned}
$$

where $m_{e}, n_{e}, \mathbf{v}_{e}$, and $\nabla p_{e}$ are the electron mass, density, velocity, and pressure gradient and $\nu_{e \alpha}$ is the Coulomb momentum-transfer frequency for scattering off species $\alpha$. This collision term is not the binary treatment used in the simulations presented here. It uses instead a distribution averaged frequency, which is given by Spitzer for a completely ionized Maxwellian distribution [56]:

$$
\nu_{\alpha \beta}=\frac{4 \sqrt{2 \pi} e^{4} Z_{\alpha}^{2} Z_{\beta}^{2} n_{\beta} \ln \Lambda_{\alpha \beta}}{\left(4 \pi \epsilon_{0}\right)^{2} 3 m_{\alpha} \mu_{\alpha \beta}}\left(\frac{T_{\alpha}}{m_{\alpha}}+\frac{T_{\beta}}{m_{\beta}}\right)^{-3 / 2}
$$

where $\mu_{\alpha \beta}=m_{\alpha} m_{\beta} /\left(m_{\alpha}+m_{\beta}\right)$ and $\ln \Lambda_{\alpha \beta}$ is the Coulomb logarithm. In the fluid model term $\mathbf{j} / \sigma$, the conductivity derives from a linearized solution for an assumed Maxwell distribution without electron inertia.

Using the generalized Ohm's law presented by Braginskii [57], $\mathbf{j}$ is expressed as the sum of components parallel and perpendicular to $\mathbf{B}$ plus a Hall term:

$$
\mathbf{j}=\sigma \mathbf{E}_{\|}^{\prime}+\sigma_{\perp} \mathbf{E}_{\perp}^{\prime}+\sigma_{\perp} \frac{\omega_{c}}{\nu_{e i}}\left(\mathbf{b} \times \mathbf{E}^{\prime}\right),
$$

where $\mathbf{b}=\mathbf{B} / B, \omega_{c}$ is the electron cyclotron frequency, $\sigma_{\perp}=\sigma /\left(1+\left(\omega_{c} / \nu_{e i}\right)^{2}\right)$, and $\nu_{e i}$ is the electron-ion collision rate from Eq. (7). The effective electric field $\mathbf{E}^{\prime}$ in the ion inertial frame is

$$
\mathbf{E}^{\prime}=\mathbf{E}+\left(\mathbf{v}_{i} \times \mathbf{B}\right)+\frac{1}{n_{e} e}\left(\nabla p_{e}-\mathbf{R}_{T}\right),
$$

where $\mathbf{R}_{T}$ is the thermal force [Ref. [57] Eq. (2.9)],

$$
\mathbf{R}_{T}=-0.71 n_{e} \nabla_{\|} T_{e}-1.5 \frac{n_{e} \nu_{e i}}{\omega_{c}}\left(\mathbf{b} \times \nabla T_{e}\right),
$$

and electron inertia and viscosity are ignored. The electron inertia may be reintroduced to the Braginskii treatment as an additional term to $\mathbf{E}_{\perp}^{\prime} \rightarrow \mathbf{E}_{\perp}^{\prime}+\left(m_{e} / e\right)\left(\partial \mathbf{v}_{e} / \partial t\right)$ where it increases frictional drag.

In coordinates relevant to the $Z$ machine, $\mathbf{B}=-B \hat{\theta}$ and Eq. (8) becomes

$$
\begin{aligned}
& j_{r}=\sigma_{\perp}\left(E_{r}^{\prime}-\frac{\omega_{c}}{\nu_{e i}} E_{z}^{\prime}\right) \\
& j_{z}=\sigma_{\perp}\left(E_{z}^{\prime}+\frac{\omega_{c}}{\nu_{e i}} E_{r}^{\prime}\right),
\end{aligned}
$$

in which Hall fields are established perpendicular to both $B_{\theta}$ and the direction of current flow. Using $\sigma=$ $n_{e} e^{2} /\left(m_{e} \nu_{e i}\right)$ to rewrite the conductivity in the Hall term,

$$
\sigma_{\perp} \frac{\omega_{c}}{\nu_{e i}}=\frac{n_{e} e^{2} \omega_{c}}{m_{e}\left(\nu_{e i}^{2}+\omega_{c}^{2}\right)},
$$

shows that in the limit $\omega_{c} \gg \nu_{e i}$, the Hall term is linear in $n_{e}$, inversely proportional to $\omega_{c}$, and independent of $T_{e}$. (It is also independent of mass, so it may be applied to ion transport).

In simulations, instabilities drive charge separation near the cathode on mm scales in $r$ and $z$, leading to very large electric field gradients, also on $\mathrm{mm}$ scales. Through an interrelated transport, these small-scale charge distributions, $j_{r, z}$, and $E_{r, z}$ propagate to mid gap. The fields and currents created are analogous to those in Eq. (9). A contour map of $E_{r}$ from the simulation in Fig. 12 is shown in Fig. 15(a). The $E_{z}$ distribution has similar mm-scale fluctuations from -20 to $20 \mathrm{MV} / \mathrm{cm}$.

The confining $B_{\theta}$ is overcome over time as the Hall fields form across the AK gap. The result is a volumetric current loss as shown in Fig. 15(b).

The relationship of current loss to $n_{e}$ more closely resembles the conductivity scaling in the Hall term in Eq. (9) [with Eq. (10)] than in $\sigma_{\perp}$. To demonstrate, we estimate the effective $\sigma$ in simulation using $I_{\text {loss }} \simeq \sigma E A$, where $I_{\text {loss }}$ is from Fig. 14, $E$ is the root-mean-square value in the loss region in Fig. 15(a), and $A$ is the area of the anode surface over which the loss occurs from Fig. 15(b). The coincident $n_{e}$ is the mean value in the loss region. This process was repeated for the four simulations plotted 

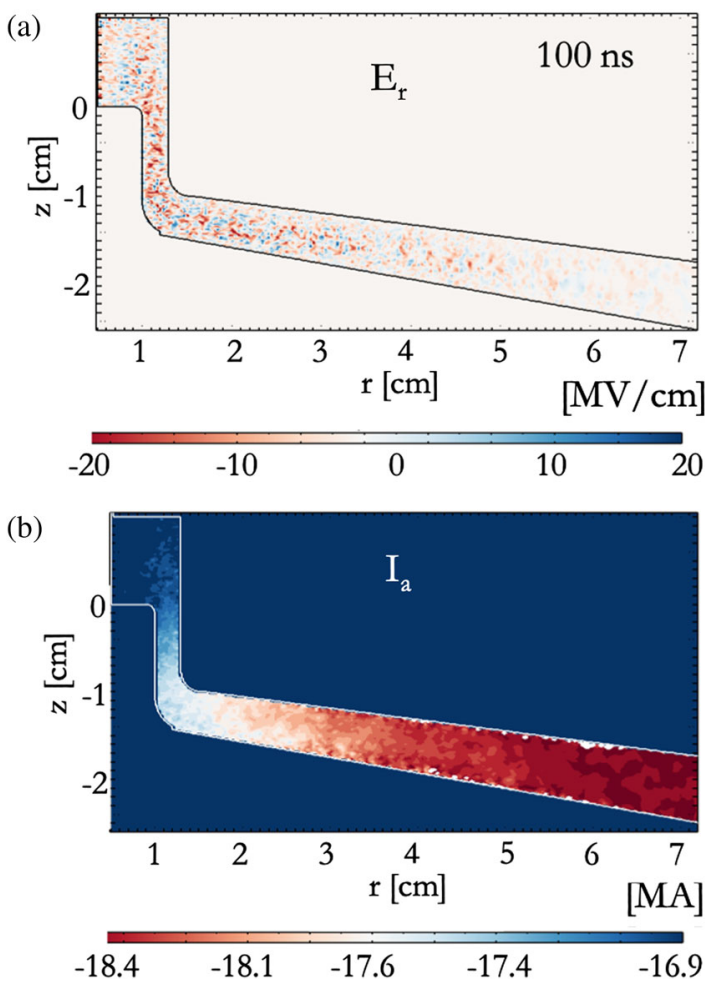

FIG. 15. Contour maps of (a) $E_{r}$ and (b) the anode current at $100 \mathrm{~ns}$ for the simulation in Fig. 12(a).

in Fig. 14. These results are compared to the fluid $\sigma_{\perp}$ and Eq. (10) in Fig. 16.

While the conductivities estimated from the kinetic simulations are nearly an order of magnitude below the OD generalized Ohm's law model, they demonstrate the same linear scaling with $n_{e}$, when $\omega_{c} \gg \nu_{e i}$ in the fluid approximation. Nonlinearities such as spatial variation in the plasma density found in the 2D and 3D kinetic simulations, and the assumption that $E^{\prime}=E$, likely contribute to the reduced effective conductivity.

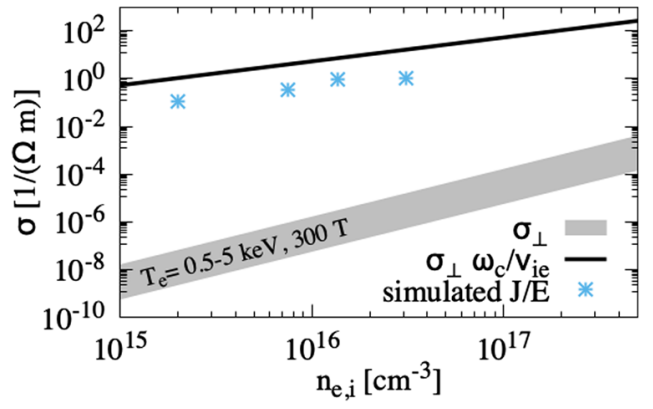

FIG. 16. The conductivity perpendicular to the $\mathbf{B}$ field as a function of $n_{e} . \sigma_{\perp}$ is plotted for the range $T_{e}=0.5-5 \mathrm{keV}$ with fixed $B=300 \mathrm{~T}$ and $n_{i}=n_{e}$ (gray). Equation (10) is plotted for $300 \mathrm{~T}$ (black). Estimates of $\sigma_{\perp}=\mathbf{J}_{\perp} / \mathbf{E}_{\perp}$ from the simulations in Fig. 14 are shown as points.

\section{SUMMARY}

Large-scale, multi-dimensional simulations of the 26-MA $Z$ machine have been conducted using the kinetic code CHICAGO. The simulations follow power flow in the $Z$ system from its four radial power feeds through its current adder (a double post-hole convolute) to the load. The physics modeled ranges from vacuum transport to chargedparticle emission and insulated flow to electrode plasma formation and expansion. In all but the vacuum stage of operation, some fraction of the current is shunted across the transmission line gaps.

Standard transport (uninsulated charged-particle flows) accounts for the cross-gap current in regions in which the current density is a few $10^{4}-10^{5} \mathrm{~A} / \mathrm{cm}^{2}$. This includes the outer feeds and both the $\varnothing 15 \mathrm{~cm}$ and the $\varnothing 31 \mathrm{~cm}$ convolutes. In the downstream portions of the convolute, electrons deposit energy along the anode posts, center, and top plates, augmenting the Joule heating. While patches of the electrode surfaces exceed the temperature threshold for contaminant desorption, the plasmas generated contribute to standard transport. Even in the $\varnothing 15 \mathrm{~cm}$ convolute, which reaches the desorption threshold midpulse, the plasma density is in the $10^{14}-\mathrm{cm}^{-3}$ range.

In regions with electrode current density $>10^{6} \mathrm{~A} / \mathrm{cm}^{2}$ $(r \leq 4.5 \mathrm{~cm})$, electrode surface plasmas form during the pulse rise and evolve velocity-shear instabilities. The instabilities give rise to $\mathrm{mm}$-scale charge separations and space-charge-generated $E$ fields. The fields reach the $\mathrm{MV} / \mathrm{cm}$ range and evolve a Hall-field-related transport which scales with electron density. These simulations indicate a plasma-conductivity-limited, rather than a space-charge-limited or magnetically insulated current loss mechanism. Thus, the current loss may be mitigated by reducing the contaminant inventory and the ratio of $E / B$. Further study of this physics will include a more granular study of particle velocities and field magnitudes. The roles of radiation transport and complex water chemistry will also be investigated.

\section{ACKNOWLEDGMENTS}

Sandia National Laboratories is a multimission laboratory managed and operated by National Technology \& Engineering Solutions of Sandia, LLC., a wholly owned subsidiary of Honeywell International, Inc., for the U.S. Department of Energy's National Nuclear Security Administration under Contract No. DE-NA0003525. The views expressed in the article do not necessarily represent the views of the U.S. Department of Energy or the United States Government. This project was partially supported by LDRD Project No. 209240. We acknowledge a great debt to the convolute design and power flow research of Drs. W. A. Stygar and T. Pointon. We thank the $Z$ operations teams and the $Z$ diagnostic teams for their support of the $Z$ facility. 
[1] M. K. Matzen, Z pinches as intense x-ray sources for highenergy density physics applications, Phys. Plasmas 4, 1519 (1997).

[2] M. G. Haines, A review of the dense Z-pinch, Plasma Phys. Controlled Fusion 53, 093001 (2011).

[3] R. B. Spielman et al., Tungsten wire-array Z-pinch experiments at 200 TW and 2 MJ, Phys. Plasmas 5, 2105 (1998).

[4] M. E. Savage, K. R. LeChien, M. R. Lopez, B. S. Stoltzfus, W. A. Stygar, D. S. Artery, J. A. Lott, and P. A. Corcoran, in 2011 IEEE Pulsed Power Conference (IEEE, 2011), pp. 983-990, https://ieeexplore.ieee.org/ stamp/stamp.jsp?tp=\&arnumber $=6191629$.

[5] D. Jianjun, X. Weiping, F. Suping, W. Meng, L. Hongtao, S. Shengyi, X. Minghe, H. An, T. Qing, G. Yuanchao, G. Yongchao, W. Bin, Z. Wenkang, H. Xianbin, W. Lijuan, Z. Zhaohui, H. Yi, and Y. Libing, Initial Performance of the primary test stand publisher: IEEE, IEEE Trans. Plasma Sci. 41, 2580 (2013).

[6] J. Deng, W. Xie, S. Feng, M. Wang, H. Li, S. Song, M. Xia, J. Ce, A. He, Q. Tian, Y. Gu, Y. Guan, B. Wei, X. Huang, X. Ren, J. Dan, J. Li, S. Zhou, H. Cai, S. Zhang, K. Wang, Q. Xu, Y. Wang, Z. Zhang, G. Wang, S. Guo, Y. He, Y. Zhou, Z. Zhang, L. Yang, and W. Zou, Matter and Radiation at Extremes 1, 48 (2016).

[7] B. A. Kablambaev, A. V. Luchinsky, V. K. Petin, N. A. Ratakhin, S. A. Sorokin, V. F. Fedushchak, S. A. Chaikovsky, and S. V. Shlyakhtun, Vopr. At. Nauki Tekhn. 34, 149 (2001).

[8] S. A. Sorokin, Formation of a pinched electron beam and an intense x-ray source in radial foil rod-pinch diodes, Phys. Plasmas 23, 043110 (2016).

[9] B. T. Hutsel, P. A. Corcoran, M. E. Cuneo, M. R. Gomez, M. H. Hess, D. D. Hinshelwood, C. A. Jennings, G. R. Laity, D. C. Lamppa, R. D. McBride, J. K. Moore, A. Myers, D. V. Rose, S. A. Slutz, W. A. Stygar, E. M. Waisman, D. R. Welch, and B. A. Whitney, Transmissionline-circuit model of an 85-TW, 25-MA pulsed-power accelerator, Phys. Rev. Accel. Beams 21, 030401 (2018).

[10] W. Zou, F. Guo, L. Chen, S. Song, M. Wang, W. Xie, and J. Deng, Full circuit calculation for electromagnetic pulse transmission in a high current facility, Phys. Rev. Accel. Beams 17, 110401 (2014).

[11] D. V. Rose, E. A. Madrid, D. R. Welch, R. E. Clark, C. B. Mostrom, W. A. Stygar, and M. E. Cuneo, Computational analysis of current-loss mechanisms in a post-hole convolute driven by magnetically insulated transmission lines, Phys. Rev. Accel. Beams 18, 030402 (2015).

[12] T. C. Genoni, R. E. Clark, and D. R. Welch, The Open Plasma Physics Journal 3, 36 (2010).

[13] D. R. Welch, N. Bennett, D. Rose, C. Thoma, and W. Stygar, Electrode contaminant plasma effects in $10^{7}-A \mathrm{Z}$ pinch accelerators, Phys. Rev. Accel. Beams 22, 070401 (2019).

[14] D. Welch, T. Genoni, R. Clark, and D. Rose, Adaptive particle management in a particle-in-cell code, J. Comput. Phys. 227, 143 (2007).

[15] N. Bruner, T. Genoni, E. Madrid, D. Rose, D. Welch, K. Hahn, J. Leckbee, S. Portillo, B. Oliver, V. Bailey, and D. Johnson, Modeling particle emission and power flow in pulsed-power driven, nonuniform transmission lines, Phys. Rev. Accel. Beams 11, 040401 (2008).
[16] N. Bennett et al., Shot reproducibility of the self-magneticpinch diode at 4.5 MV, Phys. Rev. Accel. Beams 17, 050401 (2014).

[17] I. Langmuir, The effect of space charge and residual gases on thermionic currents in high vacuum, Phys. Rev. 2, 450 (1913).

[18] M. R. Gomez, R. M. Gilgenbach, M. E. Cuneo, C. A. Jennings, R. D. McBride, E. M. Waisman, B. T. Hutsel, W. A. Stygar, D. V. Rose, and Y. Maron, Experimental study of current loss and plasma formation in the $\mathrm{Z}$ machine post-hole convolute, Phys. Rev. Accel. Beams 20, 010401 (2017).

[19] W. A. Stygar et al., 55-TW magnetically insulated transmission-line system: Design, simulations, and performance, Phys. Rev. Accel. Beams 12, 120401 (2009).

[20] D. Welch, D. Rose, B. Oliver, and R. Clark, Simulation techniques for heavy ion fusion chamber transport, Nucl. Instrum. Methods Phys. Res., Sect. A 464, 134 (2001).

[21] D. R. Welch, D. V. Rose, R. E. Clark, T. C. Genoni, and T. P. Hughes, Implementation of an non-iterative implicit electromagnetic field solver for dense plasma simulation, Comput. Phys. Commun. 164, 183 (2004).

[22] D. Rose, D. Welch, B. Oliver, R. Clark, D. Johnson, J. Maenchen, P. Menge, C. Olson, and D. Rovang, Coupled particle-in-cell and Monte Carlo transport modeling of intense radiographic sources, J. Appl. Phys. 91, 3328 (2002).

[23] D. Hinshelwood, Naval Research Laboratory, Washington, DC, Memo. Report No. 5185, 1983.

[24] M. E. Cuneo et al., Magnetically driven implosions for inertial confinement fusion at sandia national laboratories, IEEE Trans. Plasma Sci. 40, 3222 (2012).

[25] C. K. Birdsall and A. B. Langdon, Plasma Physics via Computer Simulation (Adam Hilger, New York, 1991).

[26] B. B. Godfrey, Time-biased field solver for electromagnetic codes, Mission Research Corp. Technical Report No. AMRC-N-138, 1980, presented at the 9th Conference on the Numerical Simulation of Plasmas.

[27] H. Knoepfel, Magnetic Fields (John Wiley and Sons, New York, 2000).

[28] J. Valencia and P. Quested, in ASM Handbook, Vol. 15: Castings (ASM International, Materials Park, Ohio, 2008).

[29] W. A. Stygar, S. E. Rosenthal, H. C. Ives, T. C. Wagoner, G. O. Allshouse, K. E. Androlewicz, G. L. Donovan, D. L. Fehl, M. H. Frese, T. L. Gilliland, M. F. Johnson, J. A. Mills, D. B. Reisman, P. G. Reynolds, C. S. Speas, R. B. Spielman, K. W. Struve, A. Toor, and E. M. Waisman, Energy loss to conductors operated at lineal current densities $\leq 10 \mathrm{MA} / \mathrm{cm}$ : Semianalytic model, magnetohydrodynamic, Phys. Rev. Accel. Beams 11, 120401 (2008).

[30] W. Leo, Techniques for Nuclear and Particle Physics Experiments, 2nd ed. (Springer-Verlag, New York Berlin Heidelberg, 1994).

[31] R. Fowler and L. Nordheim, Electron emission in intense electric fields, Proc. R. Soc. A 119, 173 (1928).

[32] T. W. L. Sanford, J. A. Halbleib, J. W. Poukey, A. L. Pregenzer, R. C. Pate, C. E. Heath, R. Mock, G. A. Mastin, D. C. Ghiglia, T. J. Roemer, P. W. Spence, and G. A. Proulx, Measurement of electron energy deposition necessary to 
form an anode plasma in $\mathrm{Ta}$, $\mathrm{Ti}$, and $\mathrm{C}$ for coaxial bremsstrahlung diodes, J. Appl. Phys. 66, 10 (1989).

[33] N. Bruner, D. R. Welch, K. D. Hahn, and B. V. Oliver, Anode plasma dynamics in the self-magnetic-pinch diode, Phys. Rev. Accel. Beams 14, 024401 (2011).

[34] N. Bennett, D. R. Welch, T. J. Webb, M. G. Mazarakis, M. L. Kiefer, M. D. Crain, D. W. Droemer, R. E. Gignac, M. D. Johnston, J. J. Leckbee, I. Molina, D. Nielsen, R. Obregon, T. Romero, S. Simpson, C. C. Smith, F. L. Wilkins, and D. Ziska, The impact of plasma dynamics on the self-magnetic-pinch diode impedance, Phys. Plasmas 22, 033113 (2015).

[35] M. E. Cuneo, P. R. Menge, D. Hanson, W. Fowler, M. Bernard, G. Ziska, A. Filuk, T. Pointon, R. Vesey, D. Welch, J. E. Bailey, M. Desjarlais, T. Lockner, T. Mehlhorn, S. Slutz, and M. Stark, Results of vacuum cleaning techniques on the performance of LiF fieldthreshold ion sources on extraction applied-B ion diodes at 1-10 TW, IEEE Trans. Plasma Sci. 25, 229 (1997).

[36] J. P. VanDevender, R. W. Stinnett, and R. J. Anderson, Negative ion losses in magnetically insulated vacuum gaps, Appl. Phys. Lett. 38, 229 (1981).

[37] A. A. Noyes and H. A. Wilson, The thermal ionization of gaseous elements at high temperatures, Proc. Natl. Acad. Sci. U.S.A. 8, 303 (1922).

[38] P. Redhead, The Physical Basis for Ultrahigh Vacuum (Chapman and Hall, London, 1968).

[39] M. Li and H. F. Dylla, Model for the outgassing of water from metal surfaces, J. Vac. Sci. Technol. A 11, 1702 (1993).

[40] H. F. Dylla, D. M. Manos, and P. H. LaMarche, Correlation of outgassing of stainless steel and aluminum with various surface treatments, J. Vac. Sci. Technol. A 11, 2623 (1993).

[41] J. M. D. Lane, K. Leung, A. P. Thompson, and M. E. Cuneo, Water desorption from rapidly-heated metal oxide surfacesfirst principles, molecular dynamics, and the Temkin isotherm, J. Phys. Condens. Matter 30, 465002 (2018).

[42] K. Nanbu and S. Yonemura, J. Comp. Phys. 145, 639 (1998).

[43] J. M. Creedon, Relativistic Brillouin flow in the high $v / \gamma$ diode, J. Appl. Phys. 46, 2946 (1975).

[44] C. W. Mendel and S. E. Rosenthal, Modeling magnetically insulated devices using flow impedance, Phys. Plasmas 2, 1332 (1995).

[45] J. P. Boris, Proceedings 4th Conference on Numerical Simulation of Plasmas, edited by J. Boris and R. Shanny
(Naval Research Laboratory, Washington DC, 1970), pp. 3-67.

[46] B. W. Church and R. N. Sudan, A self-consistent quasistatic equilibrium for non-neutral diamagnetic electron vortices, Phys. Plasmas 2, 1837 (1995).

[47] T. C. Wagoner et al., Differential-output B -dot and D -dot monitors for current and voltage measurements on a 20-MA, 3-MV pulsed-power accelerator, Phys. Rev. Accel. Beams 11, 100401 (2008).

[48] S. A. Slutz, M. C. Herrmann, R. A. Vesey, A. B. Sefkow, D. B. Sinars, D. C. Rovang, K. J. Peterson, and M. E. Cuneo, Pulsed-power-driven cylindrical liner implosions of laser preheated fuel magnetized with an axial field, Phys. Plasmas 17, 056303 (2010).

[49] M. R. Gomez et al., Demonstration of thermonuclear conditions in magnetized liner inertial fusion experiments, Phys. Plasmas 22, 056306 (2015).

[50] T. D. Pointon, W. A. Stygar, R. B. Spielman, H. C. Ives, and K. W. Struve, Particle-in-cell simulations of electron flow in the post-hole convolute of the $\mathrm{Z}$ accelerator, Phys. Plasmas 8, 4534 (2001).

[51] J. Swegle and E. Ott, Linear waves and instabilities on magnetically insulated gaps, Phys. Fluids 24, 1821 (1981).

[52] D. P. Chernin, Resistive wall effect on the stability of planar relativistic Brillouin flow, Phys. Fluids 25, 1342 (1982).

[53] C. L. Chang, T. M. Antonsen, E. Ott, and A. T. Drobot, Instabilities in magnetically insulated gaps with resistive electrode plasmas, Phys. Fluids 27, 2545 (1984).

[54] C. Thoma, T. C. Genoni, D. R. Welch, D. V. Rose, R. E. Clark, C. L. Miller, W. A. Stygar, and M. L. Kiefer, Numerical simulation of cathode plasma dynamics in magnetically insulated vacuum transmission lines, Phys. Plasmas 22, 032101 (2015).

[55] M. H. Hess, K. J. Peterson, D. J. Ampleford, B. T. Hutsel, C. A. Jennings, M. R. Gomez, D. H. Dolan, G. K. Robertson, S. L. Payne, W. A. Stygar, M. R. Martin, and D. B. Sinars, Design and testing of a magnetically driven implosion peak current diagnostic, Phys. Plasmas 25, 042702 (2018).

[56] P. W. Rambo and R. J. Procassini, A comparison of kinetic and multifluid simulations of laser-produced colliding plasmas, Phys. Plasmas 2, 3130 (1995).

[57] S. I. Braginskii, in Reviews of Plasma Physics, edited by M. A. Leontovich (Consultants Bureau, New York, 1965), Vol. 1, p. 205. 